\title{
Driver emotion recognition framework based on electrodermal activity measurements during simulated driving conditions
}

\begin{abstract}
An extensive variety of wellbeing frameworks had been introduced in modern vehicles a decade ago. Traction control, auto-braking, and anti-sleep systems are significant innovations that are presumed to be superior over human reaction. However, accident rates in Malaysia have yet to be fully reduced. In fact, in 2013, nearly one million enlisted vehicles were involved in road accidents, with damages reaching over RM9.3 billion. Meanwhile, a car is a system that encompasses the road, the vehicle, and the driver. At present, roads and vehicles have gained immense stability, but the driver remains as the most fragile component of this system. Electrodermal activity (EDA) was used in this study to investigate stress and anger as primary emotions leading to possible accidents involving the driver. A simulated driving assignment with preset neutral, stress, and anger scenarios was developed for emotional stimulation. A total of 20 subjects were included in this experiment. Acquired EDA signals were bandpass-filtered at $0.5 \mathrm{~Hz}$ to $2 \mathrm{~Hz}$ and subjected to short-time Fourier transform. Then, their mean, median, and variance of power spectral density were extracted. The parameters obtained were statistically analyzed with two-sample f-test. EDA readings from drivers demonstrated significant differences among neutral-stress, neutral-anger, and stress-anger simulated driving scenarios. The dataset was also divided into two groups (10-10) for training and testing of support vector machine classifier at 10 -fold cross-validation. The classification accuracy was $85 \%$ each for neutral-stress and neutral-anger and $70 \%$ for stress-anger.
\end{abstract}

Keyword: Anger; Driving; Electrodermal activity (EDA); Emotion; Galvanic skin response (GSR); Stress 